\title{
Editorial
}

\section{Cushing syndrome: reducing morbidity and mortality}

\author{
Noel P Somasundaram ${ }^{1}$, Charles N Antonypillai ${ }^{2}$, Ashley B Grossman ${ }^{3}$
}

Sri Lanka Journal of Diabetes, Endocrinology and Metabolism 2014; 4: 1-2

Despite the many advances in biochemical testing, imaging and new therapies, Cushing syndrome (CS) continues to be a challenge. Morbidity and mortality in uncured Cushing patients are high; reports suggesting a 5-year mortality of up to $50 \%$ (1). Even in those with cured CS 10-year mortality rates are higher than those without CS (2,3). The key morbidities include opportunistic infection in the short-term and cardiovascular complications in the long-term. A comprehensive strategy of diagnosing urgently, identifying co-morbidities and treating them early, referring to a specialized center appropriately and offering treatment rapidly should decrease the morbidity among patients. It is very appropriate that in this issue of the Journal, the Endocrine Society of Sri Lanka has published the new guidelines on the management of CS. We trust that the clinicians will find clearer directions in managing patients with CS. As in any complex disorder, patients with CS should be comprehensively and rapidly managed with the application of a systematic approach to enable rapid diagnosis leading to early treatment of CS.

Some specialized endocrine centers employ a twoweek rule in the management of Cushing syndrome. The two-week rule dictates aggressive investigation to localize the source of Cushing syndrome and, if a diagnosis is not made within a period of two-weeks, the patient is encouraged to undergo bilateral adrenalectomy and testing is continued afterwards. Although not all units are capable of performing all the tests necessary within a short period of time, the principle illustrates the urgency and the lifethreatening nature of the illness and is a sober reminder to act with more aggressive intent in the management. It may be preferable in the most severe cases to truncate investigation while medical therapy to lower cortisol levels is undertaken, rather than proceeding to bilateral adrenalectomy in a sick immunocompromised patient who is also at high risk of venous thrombo-embolism. Hypercortisolic patients are immunosuppressed and are susceptible to opportunistic infections, including
Aspergillus species, Cryptoccus neoformans, Pneumocystis carinii, Nocardia asteroids, Staphylococcus aureus, Candida albicans, and Herpes simplex (4).

The guidelines by the Endocrine Society of Sri Lanka emphasize the need to employ two screening tests to confirm the diagnosis of Cushing syndrome and early referral to a specialized centre for diagnosing the cause. The initial screening tests either demonstrate disruption of the usual cortisol hemodynamics or an increased cortisol level, and include overnight and low-dose dexamethasone suppression tests, measurement of midnight cortisol and urine free cortisol. In a world where obesity is becoming a common problem, using appropriate clinical skills to select patients who have subtle and early features of Cushing syndrome will be essential. Once Cushing syndrome is diagnosed, measurement of ACTH is used for etiological classification as ACTH-dependent or ACTH-independent Cushing syndrome. Further investigations usually imaging followed by catheter studies and other tests such as CRH or desmopressin stimulation tests would be sufficient in most patients. The difficult cases are the ones where CS caused by a small ectopic lesion that is not immediately apparent.

The treatment of choice is surgery to remove the source of the lesion. A short period of medical therapy to make the patient eucortisolic in order to decrease the metabolic derangements may be necessary before surgery to optimize surgical outcomes, including wound healing. In the patient who is cured or in remission after surgery, continued surveillance is necessary to manage the suppressed pituitary-adrenal axis and to detect recurrence early. One of the key reasons for early mortality in the patient cured of CS is the suppressed hypothalamopituitary-adrenal axis: it is imperative to offer the patient appropriate glucocorticoid support necessary for daily living and for emergencies. Long term morbidity and mortality reduction should be achieved by aggressive cardiovascular risk factor control, although data are lacking at the moment to this approach.

${ }^{1}$ Diabetes and Endocrine Unit, National Hospital of Sri Lanka, Colombo, Sri Lanka, ${ }^{2}$ Diabetes and Endocrine Unit, Teaching Hospital, Kandy, Sri Lanka, ${ }^{3}$ Oxford Centre for Diabetes, Endocrinology and Metabolism, OCDEM, University of Oxford, UK. 


\section{References}

1. Plotz D, Knowlton AI, Ragan C. The natural history of Cushing's disease. Am J Med 1952; 13: 597-614.

2. Ntali G, Asimakopoulou A, Siamatras T, et al. Mortality in Cushing's syndrome: systematic analysis of a large series with prolonged follow-up. Eur J Endocrinol 2013; 169(5):715-23
3. Yaneva M, Kalinov K, Zacharieva S. Mortality in Cushing's syndrome: data from 386 patients from a single tertiary referral center Eur J Endocrinol 2013;169(5): 621-7.

4. Bakker RC, Gallas PR, Romijn JA, Wiersinga WM. Cushing's syndrome complicated by multiple opportunistic infections. J Endocrinol Invest 1998; 21(5): 329-33. 